\title{
Chitosan and its composites: Properties for use in bone substitution
}

\author{
Marek Stępniewski ${ }^{A-D}$, Jacek Martynkiewicz ${ }^{A-D}$, Jerzy Gosk ${ }^{A-C, E, F}$ \\ Clinical Department of Traumatology and Hand Surgery, Wroclaw Medical University, Wrocław, Poland \\ A - research concept and design; $\mathrm{B}$ - collection and/or assembly of data; $\mathrm{C}$ - data analysis and interpretation; \\ $D$ - writing the article; $E$ - critical revision of the article; $F$ - final approval of article
}

Polymers in Medicine, ISSN 0370-0747 (print), ISSN 2451-2699 (online)

Polim Med. 2017;47(1):49-53

\section{Address for correspondence \\ Marek Stępniewski \\ E-mail: marek.stepniewski@wp.pl}

\section{Funding sources}

none declared

\section{Conflict of interest}

none declared

Received on July 18, 2017

Revised on August 06, 2017

Accepted on August 20, 2017

\begin{abstract}
For many years, research has been carried out on finding an ideal bone substitute. Chitosan (CTS) is a naturally occurring polysaccharide, obtained mainly from, inter alia, the shells of crustaceans. It is characterized by its high level of biocompatibility, biodegradability and antimicrobial properties as well as its support in the healing of wounds. Chitosan, due to its ability to form porous structures, can be used in the production of scaffolds used in the treatment of bone defects. There are numerous studies on the use of CTS in combination with other substances which aim to improve its biological and mechanical properties.

The combination of chitosan with the calcium phosphate hydroxyapatite (HAp) has been extensively tested. The objective of the current studies is to verify the properties of scaffolds consisting of chitosan and other substances like polybutylene succinate, human bone marrow mesenchymal stem cells (hBMSCs), collagen, alginate, transforming growth factor - $\beta$ (TGF- $\beta$ ), insulin-like growth factor (IGF), platelet-derived growth factor (PDGF) or bone morphogenetic proteins (BMP). The aim of the current research is to develop a scaffold with sufficiently good mechanical properties. Trials are underway with many of the biological and synthetic components affecting the biological properties of chitosan. This will allow for the creation of a substitute that fully meets the conditions for an ideal artificial bone.
\end{abstract}

Key words: chitosan, chitin, scaffold, bone substitution
DOI

10.17219/pim/76517

\section{Copyright}

@ 2017 by Wroclaw Medical University

This is an article distributed under the terms of the Creative Commons Attribution Non-Commercial License (http://creativecommons.org/licenses/by-nc-nd/4.0/) 
The ideal material used to supplement bone defects should possess 4 properties: osteoconduction, osteoinduction, osteointegration and osteogenesis. Osteoconduction is the ability of a material to promote bone growth by creating appropriate physical conditions, such as the porous spatial structure through which osteogenic or neoplastic cells penetrate the bone. Osteoinduction involves the exertion of influence on stem cells, targeting them to differentiate into osteoblasts, which are bone-forming cells. The most active group of osteoinductive factors are bone morphogenetic proteins (BMP). Osteointegration is the direct bonding of a new living bone with the bone substitute and as well as the gradual replacement of the substitute with living bone. Osteogenesis is the formation of new bone by osteogenic cells. ${ }^{1}$

Osteointegration is a particularly important attribute of a graft, which significantly influences the final effect. Usually, this process is slow and does not always complete the graft remodeling process. Many factors affect the effectiveness of this joining in which the graft-forming material plays a crucial role, possessing properties of porosity and affecting the quality of the bone at the location of the graft's implantation.

There are several phases in the grafting process. In the initial phase, the transplant is filled with flowing blood on which basis the hematoma develops. Inflammatory cells and growth factors are introduced through this blood flow. Then there is a dilatation phase, in which, by the formation of new blood vessels and connective tissue, the graft initially attaches to the bone tissue. In subsequent stages the osteoclasts resorb elements of the graft where, in place of the osteocytes, new bone is formed, connecting both elements permanently. The last phase is the remodeling of the newly formed bone, leading to the rebuilding of the natural character of bones.

In orthopedics, 3 types of transplants are used for the repair of bone defects: autographs, allografts and bone substitutes. The reconstruction process is most effective in an autograft, i.e. bone graft. Its structure is porous, allowing easy penetration of new bone cells as well as new vessels. Autograft cells are mostly covered by osteoblasts that exhibit traits of osteoconductivity and osteoinductance. The most common graft location is the iliac crest. This is a perfect graft, but due to the limited amount of tissue available, the pain involved, the possibility of infection and increased blood loss or prolonged surgery time, other forms of bone substitution are also used. ${ }^{2}$

The most common alternative to self-bone grafting is allografts, i.e. grafts of material from a deceased donor. They can be prepared in any shape or size. However, besides providing the spatial structure of grafts, they do not meet the requirements of osteoinductivity, which may result in a weakened osteointegration. ${ }^{3}$

Due to the limitations of natural bone grafts, synthetic bone substitutes have been introduced.
Bone substitutes can be divided into 3 generations, depending on the degree of integration by the recipient's bone. The first generation includes pure metals (stainless steel, titanium), metal alloys (aluminum, zirconium) and polymers (silicone, polypropylene, polymethyl methacrylate). This group of grafts often develops a fibrous layer on the bone contact surface, which may lead to a lack of full osteointegration and a secondary loosening of the graft. The second generation of substitutes is coated with an additional supporting layer to prevent the formation and deposition of connective tissue on the graph and thereby facilitating complete osteointegration. This group includes hydroxyapatite, calcium metaphosphate and bioactive glass. The third generation uses a material most closely related to the natural structure of bone and is characterized by high osteoconductivity and bioactivity (osteoinductive and biodegradable). ${ }^{4}$

\section{Properties of bone substitutes}

One of the important properties of bone substitutes is biocompatibility. Fully biocompatible materials, i.e. materials that do not cause any bodily inflammatory reaction, either local or general, are used during the synthesis of these bone substitutes.

The porosity of the preparation is also very important. The greater the porosity, the easier it is for the new cells to penetrate. At the same time, the nature of the structure influences its mechanical strength: namely the greater the porosity, the lower the strength. The individual mechanical needs should be taken into account while preparing bone substitutes. One of the solutions is the use of "micro \& nano" technology, which allows for the graft to have a structure with different pore diameters, simultaneously providing good osteointegration and mechanical strength.,6

The biodegradability of the bone substitute material must go hand in hand with maintaining the mechanical properties of the substitute, which means that it cannot be too rapid. Degradation of the graft before complete bone remodeling can lead to a weakened structure and consequent fractures within the graft. The risk of fractures can be reduced by strengthening the osteoinduction. To achieve this, the substitute is combined with a progenitor and osteoblast stimulating agents, which lead to an increased production of bone matrix. Among other things, the following are used: transforming growth factor $-\beta$ (TGF- $\beta$ ), insulin-like growth factor (IGF), platelet-derived growth factor (PDGF), and bone morphogenetic proteins (BMP). ${ }^{6}$

\section{Chitosan}

Chitin is one of the most common polysaccharides found in nature. It occurs naturally in arthropods, sponges and corals, as well as in other organisms. Its discoverer, 
H. Brancota, was responsible for isolating chitin for the first time in 1811. The deacetylated form of chitin, called chitosan, was described by Rouget in 1859 .

For industrial purposes, chitin is mainly obtained from marine invertebrates such as crabs, shrimp or krill by a process of chemical hydrolysis. The first stage is demineralization, i.e. shredding of crustacean armor as well as $\mathrm{CaCO}_{3}$ deprivation (under the influence of $\mathrm{HCl}$ ). The next step is deproteinization with a $\mathrm{NaOH}$ solution and discoloring with a $\mathrm{KMnO}_{4}$ solution. This is how chitosan is obtained from the chitin that has undergone the deacetylation process. Alternatively, there is an enzymatic hydrolysis process using, inter alia, lysozymes or neutral proteases. ${ }^{7}$ The degree of deacetylation and molecular weight of chitosan depend on the substrate used as well as the method of production. The enzymatic method allows CTS to have higher molecular weights than other traditional methods. From the Scopulariopsis brevicaulis mushroom, the average CTS weight using the enzymatic method is $267.97 \mathrm{kDa}$ while the traditional chemical method yields $84.04 \mathrm{kDa}{ }^{7}$

\section{Properties of chitosan}

Because of its biological properties and the constantly renewable natural resource chitosan, in the form of chitin, has been a subject of scientific research for over 30 years in the field of medicine.

Chitosan exhibits several important bio-material properties. It is biocompatible, biodegradable, hydrophilic and non-toxic. It has hemostatic properties. Its porous structure facilitates penetration and binding to other cells, especially bone cells. It affects cells involved in the bone-forming process by activating macrophages and stimulating fibroblasts, and it also captures and binds to growth agents as well as supports the processes of angiogenesis. ${ }^{8,9}$ Chitosan has a cationic nature, ${ }^{10}$ which allows it to bind to negatively charged molecules, such as erythrocytes or thrombocytes, activating an extrinsic coagulation pathway. ${ }^{11}$ Chitosan also exhibits antimicrobial properties. The cationic nature makes it possible to connect with the walls of Staphylococcus aureus species. This leads to damage as well as to the inhibition of the mRNA bacteria's synthesis. It may also be a carrier for various therapeutic substances. It is used as a carrier of silver ions in the production of modern wound dressings. ${ }^{12,13}$

The ability to synthesize chitosan in various forms, i.e. paste membranes, sponges, fibers and spatial porous structures, such as a scaffold, is especially utilized in orthopedics. ${ }^{14,15}$ When supplementing bone defects, the most significant effect is through the use of spatial graphs obtained from chitosan. ${ }^{16,17}$

\section{Composite chitosan-hydroxyapatite}

Insulated chitosan does not meet all the requirements of the ideal graft, but when mixed with other composites, it comes closer to having the properties of bone.

The combination of chitosan with the calcium phosphate hydroxyapatite (HAp) has been extensively tested. HAp is one of the most stable forms of calcium phosphate. It occurs naturally in the bones, making up 60-65\% of inorganic bone components. In orthopedics, HAp has been applied as a coating for metal implants (e.g. hip endoprosthesis). The bone in contact with the HAp-coated implant begins to grow and gradually penetrates the pores, improving the mechanical attachment of the implant. HAp is also used in the supplementation of bone defects. Due to its poor mechanical properties, the grafts are limited to small sizes. ${ }^{18}$

CTS/HAp composites are completely non-toxic and exhibit a significant increase in osteoblast activity and their deposition/penetration of the composite. ${ }^{19-22}$ There are many methods for the synthesis of CTS/HAp, ranging from the simple mixing of natural HAp with CTS by means of freezing, lyophilization and hybridization as well as the use of state-of-the-art methods such as electrospinning. The method used affects the quality, especially the mechanical strength of the resulting scaffold, which depends on the bond of $-\mathrm{NH}_{2}$ and $-\mathrm{OH}$ groups of chitosan with $\mathrm{Ca}^{2+}$ hydroxyapatite and the ratio of CTS to Hap. ${ }^{20,23}$

CTS/HAp composites are characterized by significantly better mechanical properties than both products individually. The highest compression strength of the CTS/HAp scaffold, $119.86 \mathrm{MPa}$, was obtained by the use of 30:70 CTS:HAp. ${ }^{24}$ Joining of the CTS reduces the compression strength of the graft and the higher the molecular weight of the chitosan the higher the compressive strength. ${ }^{25}$

Increasing the HAp content increases the compression strength. This is related to a lower number of bonds between CTS and Hap. ${ }^{26}$ The temperature at which the synthesis of the molecules occurs, the higher the temperature, the stronger the binding, is also significant. The aquatic environment affects the decrease in mechanical properties. HAp/CTS/composite carboxymethylcellulose $(40 \% / 30 \% / 30 \%)$, is characterized by a compression strength of $40 \mathrm{MPa}$ under dry conditions and $12 \mathrm{MPa}$ in an aqueous medium. ${ }^{27}$

CTS/HAp scaffolds are characterized by their pore structure, averaging 100-200 $\mu \mathrm{m}$, while allowing free deposition and migration of osteoblasts (averaging 10-30 $\mu \mathrm{m}$ ) deep into the composite. It has been observed that osteoblasts embedded in the CTS/HAp scaffolds are activated after $30 \mathrm{~min}$ and after 5 days they will start to aggregate the bone reconstruction process. ${ }^{28}$ 
Kawakami et al. studied the in vivo effect of CTS/HAp self-hardening paste through its application on the surface after removal of the periosteum. New bone formation was observed after 1 week and continued during a 20-week follow-up. This study confirmed the osteoconductivity of chitosan. ${ }^{35}$

To assess the biological activity of the scaffolds, a level of alkaline phosphatase activity (FA) is used, which increases with increased osteoblast differentiation as well as during the early stage of bone formation. CTS/HAp composite produces a significantly higher FA growth activity than chitosan on its own. The highest FA activity was observed in composites containing 30-40\% HAp. The use of grafts with a lower HAp content was linked with a lower FA activity. ${ }^{28,29}$

\section{Other composites containing chitosan}

In order to improve the biological properties of chitosan, other associations have also been used, e.g. polybutylene succinate and bone marrow stem cells. Human bone marrow mesenchymal stem cells (hBMSCs) accelerate the rate of bone formation. Costa Pinto et al. investigated the effect of chitosan-based scaffold cultured with hBMSCs on the surrounding bone. Eight weeks after implantation in the location of the cranial defect in the skull coatings of nude mice, grafts were collected for mikroCT testing. Very good integration with the surrounding tissue as well as significant bone formation was observed. ${ }^{30}$

Chitosan composites containing collagen and $\beta$-glycerophosphate are 3 times more rigid than pure chitosan. The osteogenic properties of such scaffolds are also better. ${ }^{31}$ Adding of alginate increases the activity of osteoblasts. ${ }^{32}$ CTS/HAp composite mixed with RGD peptide (ARG-GLY-ASP) has a stronger osteoconductivity. ${ }^{33}$

Research is also being conducted on the possibility of using various scaffold growth agents. Osteogenic activity was greatly increased by insulin-like growth agent-1 (IGF-1), with slightly less bone morphogenetic protein-2 (BMP-2). Nande et al. investigated and compared the effectiveness of porous chitosan, alone and in combination with IGF-1 and BMP-2 in the healing of rabbit tibias with bone defects. Radiologically, evidence of radiodensity in the bone defect area was observed after the $60^{\text {th }}$ day (started on the $30^{\text {th }}$ day) in the rabbit group with IGF-1 and BMP-2 and in the $90^{\text {th }}$ day in the chitosan-only group. Histological observation depicted better osteoblastic proliferation, vascularization and reticular network in the group with IGF-1. ${ }^{34}$

Also, the addition of platelet-rich plasma (PRP) to the final scaffold was beneficial for osteogenesis. Bi et al. injected tricalcium phosphate/chitosan, in combination with autologous platelet-rich plasma, into the tibial bone defect of a goat. After 16 weeks, complete bone regeneration was observed. ${ }^{32}$

\section{Conclusions}

The results of many studies carried out on bone substitutes show promising results on the safety and efficacy of chitosan-based scaffolds. Compound composites of chitosan and biocompatible polymers or bioresorbable ceramics may in the future fulfil the requirements of the ideal artificial bone graft. CTS/HAp composites are characterized by good osteoconduction, osteoinductivity and stimulation of osteogenesis. The aim of current research is to develop a scaffold with sufficiently good mechanical properties. Trials are underway with many of the biological and synthetic components affecting the biological properties of chitosan. This will allow for the creation of a substitute that fully meets the conditions for an ideal artificial bone.

\section{References}

1. Greenwald AS, et al. Bone-graft substitutes: facts, fictions, and applications. J Bone Joint Surg Am. 2001;83-A Suppl 2 Pt 2:98-103.

2. Stevens B, Yang Y, Mohandas A, Stucker B, Nguyen KT. A review of materials, fabrication methods, and strategies used to enhance bone regeneration in engineered bone tissues. $J$ Biomed Mater Res B. 2008;85(2):573-582.

3. Moore WR, Graves SE, Bain GI. Synthetic bone graft substitutes. ANZ J Surg. 2001;71(6): 354-361.

4. Amini AR, Laurencin CT, Nukavarapu SP. Bone tissue engineering: recent advances and challenges. Crit Rev Biomed Eng. 2012;40(5):363-408.

5. Polo-Corrales L, Latorre-Esteves M, Ramirez-Vick JE. Scaffold Design for Bone Regeneration. J Nanosci Nanotechnol. 2014;14(1):15-56.

6. Rivera-Chacon DM, et al. Fibronectin and vitronectin promote human fetal osteoblast cell attachment and proliferation on nanoporous titanium surfaces. J Biomed Nanotechnol. 2013;9(6):1092-1097

7. Cai J, et al. Enzymatic preparation of chitosan from the waste Aspergillus niger mycelium of citric acid production plant. Carbohydr Polymers. 2006;64:151-157.

8. Muzzarelli RAA. Chitins and chitosan for the repair of wound skin, nerve, cartilage and bone. Carbohydr Polym. 2009:76(2):167-182.

9. Dai T, Tanaka M, Huang YY, Hamblin MR. Chitosan preparations for wounds and burns: antimicrobial and wound healing effects. Expert Rev Anti Infect Ther. 2011;9(7):857-879.

10. Yang TL. Chitin - based materials in tissue engineering: Applications in soft tissue and epithelial organ. Int J Mol Sci. 2011;12(3):1936-1963.

11. Cheung RCF, Ng TB, Wong JH, Chan WY. Chitosan: An update on potential biomedical and pharmaceutical applications. Mar Drugs. 2015;13(8):5156-5186.

12. Guo R, Xu S, Ma L, Huang A, Gao C. Enhanced angiogenesis of geneactivated dermal equivalent for treatment of full thickness incisional wounds in a porcine model. Biomaterials. 2010;31(28):7308-7320.

13. Liu X, Ma L, Liang J, Zhang B, Teng J, Gao C. RNAi functionalized collagen-chitosan/silicone membrane bilayer dermal equivalent for full thickness skin regeneration with inhibited scarring. Biomaterials. 2013;34(8):2038-2048.

14. Muzzarelli RA, et al. Osteoconductive properties of methylpyrrolidinone chitosan in an animal model. Biomaterials. 1993;14(12):925-929.

15. Muzzarelli RA, et al. Stimulatory effect on bone formation exerted by a modified chitosan. Biomaterials. 1994;15(13):1075-1081.

16. Costa-Pinto $A R$, et al. Osteogenic differentiation of human bone marrow mesenchymal stem cells seeded on melt based chitosan scaffolds for bone tissue engineering applications. Biomacromolecules. 2009;10(8):2067-2073.

17. Tuzlakoglu K, Alves CM, Mano JF, Reis RL. Production and characterization of chitosan fibers and 3-D fiber mesh scaffolds for tissue engineering applications. Macromol Biosci. 2004;4(8):811-819.

18. Kim SK, Mendis E. Bioactive compounds from marine processing byproducts - A review. Food Res Int. 2006;39(4):383-393. 
19. Di Martino A, Sittinger M, Risbud MV. Chitosan: A versatile biopolymer for orthopaedic tissue-engineering. Biomaterials. 2005;26(30):5983-5990.

20. Teng SH, Lee EJ, Yoon BH, Shin DS, Kim HE, Oh JS. Chitosan/nanohydroxyapatite composite membranes via dynamic filtration for guided bone regeneration. J Biomed Mater Res A. 2009;88(3):569-580.

21. Thein-Han W, Misra R. Biomimetic chitosan - nanohydroxyapatite composite scaffolds for bone tissue engineering. Acta Biomater. 2009;5(4):1182-1197.

22. Xianmiao C, Yubao L, Yi Z, Li Z, Jidong L, Huanan W. Properties and in vitro biological evaluation of nano-hydroxyapatite/chitosan membranes for bone guided regeneration. Mater Sci Eng C. 2009;29(1):29-35.

23. Yamaguchi I, et al. Preparation and microstructure analysis of chitosan/hydroxyapatite nanocomposites. J Biomed Mater Res. 2001;55(1):20-27.

24. Li Z, Yubao L, Aiping Y, Xuelin P, Xuejiang W, Xiang Z. Preparation and in vitro investigation of chitosan/nano-hydroxyapatite composite used as bone substitute materials. J Mater Sci Mater Med. 2005;16(3):213-219.

25. Li Z, Yubao L, Aiping Y, Xuelin P, Xuejiang W, Xiang Z. Bioabsorbable chitosan/hydroxyapatite composite rod for internal fixation of bone fracture prepared by in situ precipitation. Acta Polym. Sin. 2002;6:828-833.

26. Liuyun J, Yubao L, Chengdong X. A novel composite membrane of chitosan-carboxymethyl cellulose polyelectrolyte complex membrane filled with nano-hydroxyapatite I. Preparation and properties. J Mater Sci Mater Med. 2009;20(8):1645-1652.

27. Aronow MA, Gerstenfeld LC, Owen TA, Tassinari MS, Stein GS, Lian JB. Factors that promote progressive development of the osteoblast phenotype in cultured fetal rat calvaria cells. J Cell Physiol. 1990;143(2):213-221.

28. Zhang Y, Ni M, Zhang M, Ratner B. Calcium phosphate-chitosan composite scaffolds for bone tissue engineering. Tissue Eng. 2003;9(2):337-345.

29. Mukherjee DP, Tunkle AS, Roberts RA, Clavenna A, Rogers S, Smith D. An animal evaluation of a paste of chitosan glutamate and hydroxyapatite as a synthetic bone graft material. J Biomed Mater Res B Appl Biomater. 2003;67(1):603-609.

30. Costa-Pinto AR, et al. Chitosan-poly(butylene succinate) scaffolds and human bone marrow stromal cells induce bone repair in a mouse calvaria model. J Tissue Eng Regen Med. 2012;6(1):21-28.

31. Hoemann $C D$, et al. Cytocompatible gel formation of chitosanglycerol phosphate solutions supplemented with hydroxyl ethyl cellulose is due to the presence of glyoxal. J Biomed Mater Res A. 2007;83(2):521-529.

32. Bi L, Cheng W, Fan H, Pei G. Reconstruction of goat tibial defects using an injectable tricalcium phosphate/chitosan in combination with autologous platelet-rich plasma. Biomaterials. 2010;31(12):3201-3211.

33. Qu Z, Yan J, Li B, Zhuang J, Huang Y. Improving bone marrow stromal cell attachment on chitosan/hydroxyapatite scaffolds by an immobilized RGD peptide. Biomed Mater. 2010;5(6):065001.

34. Nandi SK, Kundu B, Basu D. Protein growth factors loaded highly porous chitosan scaffold: a comparison of bone healing properties. Mater Sci Eng C Mater Biol Appl. 2013;33(3):1267-1275.

35. Kawakami T, Antoh M, Hasegawa H, Yamagishi T, Ito M, Eda S. Experimental study on osteoconductive properties of a chitosan-bonded hydroxyapatite self-hardening paste. Biomaterials. 1992;13(11):759-763. 
